\title{
Automatic localization of phoenix by satellite image analysis
}

\author{
R. Cousin and M. Ferry \\ Phoenix Research Station, Spain, email: Raphael.cousin90@gmail.com
}

\begin{abstract}
Cousin, R. and M. Ferry. 2019. Automatic localization of phoenix by satellite image analysis. Arab Journal of Plant Protection, 37(2): 83-88.

The Red palm weevil (RPW) Rhynchophorus ferrugineus is becoming one of the deadliest pests of the palms in the world. In order to effectively implement a RPW control programme to achieve rapid regression of this pest, it is necessary to have GPS coordinates of each palm present on the control perimeter. This location makes it possible to establish maps and databases which are essential for organizing, at the local and national level, the implementation and permanent monitoring of control measures. It is difficult, time-consuming and expensive to locate palms by visually exploring the entire perimeter from the ground. In the zone of regular plantations, this work can be processed but it becomes extremely heavy in the traditional oasis like in urban environment where the distribution of the palms is very irregular. With advances in satellite imagery, it is possible to acquire high quality images at very short intervals of time from a standard format for a large part of the earth. Combined with the progress of machine learning, particularly deep learning, this amount of data is able to feed a robust model. It would allow to automate the detection of palms at large scale and monitor their evolution at very short intervals, which in the fight against RPW is valuable information. This first work wants to test the interest in this solution. We build and train a convolution neural network in order to find two species of palms Phoenix canariensis and Phoenix dactylifera (C\&D) in a very heterogeneous area of $100 \mathrm{~km}^{2}$. Our model evaluation shows that $1 / 5$ of the objects found are false positive and more than $2 / 3$ of $C \& D$ are perfectly localized. These first results could be improved greatly by implementing a more robust algorithm using more data and using larger colour spectrum (as near infra-red). The question of the infested palms detection using satellite imagery and machine learning stays open.
\end{abstract}

Keywords: Rhynchophorus ferrugineus, remote sensing, machine learning, convolution neural network, vision.

\section{Introduction}

The red palm weevil (RPW) Rhynchophorus ferrugineus is one of the deadliest pests of the palms in the world (FAO 2017).

When a new infested palms is found or when a RPW is caught for the first time in a trap far from any known infested palms, it is necessary to implement immediately a contingency plan after delimitation of the potentially infested area (Figure 1). This plan must include in the delimited area the following activities (Ferry et Aldobai, 2017): location of all the palms owners to alert them; inspection of all the palms to locate the palm at the origin of the new infestation; immediate sanitation or eradication of the infested palms; preventive treatments on all the palms; collocation of traps for mass trapping. The awareness of the palms owners and their training will, generally be implemented. The technical activities will be maintained till no more detection of infested palms and no more captures. The efficiency of the plan will be controlled by following permanently the evolution of the number of new infested palms and of the captures in the traps.

Such contingency plan must be implemented in all the infested areas at the local, regional and national levels. At these different scales, it is necessary to have the geolocation of all the palms. This geolocation and, consequently the information of the number of palms at each scale, will permit first to establish the programme to obtain the rapid decline of the RPW and the means need to be to dedicated for it. Then it will allow management and control of the programme and all the components of the IPM strategy with the assistance of GIS (Ferry, 2017). As emphasized by Fajardo during the Rome meeting (Fajardo, 2017), the success of the eradication programme in the Canary Islands would not have been possible without the assistance of GIS for the management of the action plan (Fajardo, 2017).

The information on the geolocation of the palms is usually not available. The geolocation of the palms by field survey constitutes an important work that require human resources, equipment and time, when action has to initiated as soon as possible. As in most of the countries, the epidemic today is very rapid, the apparition and the extension of a great number of outbreaks imply to locate the palms very quickly and on an increasing surface area. Furthermore, in many places, like the traditional oasis or the cities, the environment is very heterogeneous, therefore the localization of the palms is very complicated.

Satellite imagery continues to progress rapidly. It exists through a wide range of providers, with different spectra and resolutions for multiple usages. For example: Copernicus (European Space Agency) offers free and open source data. The two satellites Sentinel-2A (launched in 2015) and Sentinel-2B (launched in 2017) provide images of the Earth every 5 days in 13 spectral bands with a resolution of 10 to $60 \mathrm{~m}$. DigitalGlobe (American company) markets high resolution images. One of its WorldView-3 satellites (launched in 2014) provides images up to $30 \mathrm{~cm}$ per pixel for the panchromatic band. 


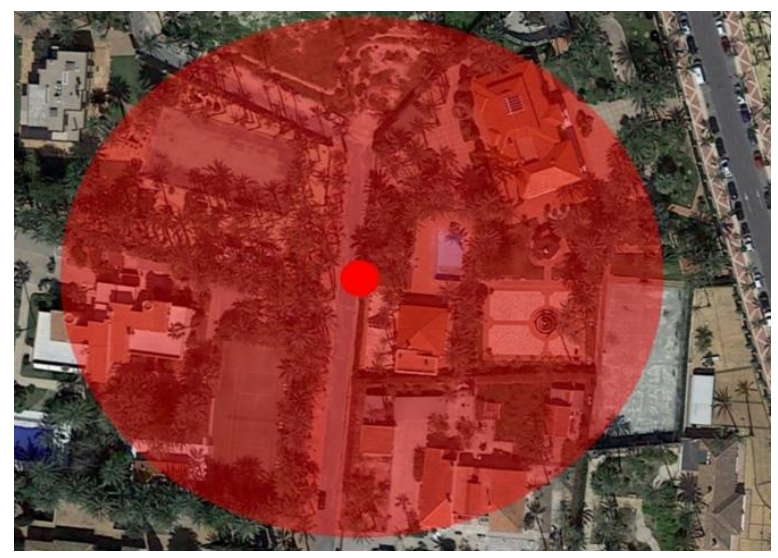

Figure 1. Illustration of the perimeter of control around infested palm tree.

At this level of resolution, it is possible to recognize by visual observation of the images, the palms with a good degree of certainty. Nevertheless, for large areas, such method of detection would represent very heavy task and the risk of error would become increasingly important.

Research has already been done using satellite images in order to automate the mapping of palm groves and the estimation of the number of palms. The analysis of the images and the contextual classification make it possible to estimate the number of the date palms with good precision using the data of WorldView 2. (Labrador-García et al., 2013).

Machine learning has achieved important progress these last years. Particularly for object detection and image classification. When the data are of sufficient quality and number, it is possible to train a model with numerous parameters such as deep convolution neural networks (deep CNN) (Krizhevsky, 2012; Simonyan, 2014). These types of classifier are able to model complex problems in which they perform better than the other approaches (LeCun, 2015).

This deep learning approach was used on satellite imagery and has already demonstrated its ability to detect and localize oil palms under homogeneous and aligned planting conditions (Li et al., 2016 and Santoso, 2016). It remains to test deep learning approach on more heterogeneous areas, such as in oases and urban areas, where RPW is more difficult to detect and monitor. It is in this sense that we conducted this first study to test the effectiveness of palms detection on satellite images.

\section{Adult weevils in the test plantations}

Pheromone trap densities in area-wide RPW-IPM programs range from one to 10 traps per hectare (Faleiro et al., 2011; Oehlschlager 2006; Soroker et al., 2005). However, increasing the trap density is often not possible due to the periodic servicing (change of food bait and water), necessary to sustain the trapping efficiency. In this context, the 'trap and bait free' A\&K option could significantly augment the mass trapping programme of RPW by killing the emerging adult weevil population in the field. A\&K technology can successfully eliminate adult RPW population. The technology serves as an excellent tool to manage RPW population where infestations are high, or in plantations that are inaccessible and neglected and could significantly strengthen the on-going pheromone trap based RPW-IPM strategy particularly in plantations where the pheromone trap density has to be increased to effectively mass trap the adult population. The cost involved in the periodic servicing associated with the traditional food-baited pheromone traps is effectively eliminated with this 'bait and trap free' technique of controlling the adult RPW population in the field. However, a minimum of one food baited pheromone trap/ha is required to be maintained in an area-wide RPW control programme to gauge weevil activity in the field. Furthermore, A\&K has the potential to do away with the need to take up routine periodic preventive insecticide sprays. The technique has been used in RPW-IPM programs in date palm, the canary palm and coconut in Mauritania, Abkhazia in the Republic of Georgia and Malaysia, respectively.

All safety precautions (wearing of gloves, mask, foot wear, etc.) need to be complied with while applying RPW $A \& \mathrm{~K}$ formulation in the field. In case of allergic reaction or coming in direct contact with the product, further application should be stopped, immediate medical assistance sought and the manufacturer contacted.

The Electrap ${ }^{\mathrm{TM}}$ is an efficient service free semiochemical mediated technology against RPW and can be incorporated in RPW-IPM programs. However, known RPW repellents need further testing to be incorporated in an RPW-IPM program involving a push-pull strategy.

\section{Materials and Methods}

\section{Area of interest}

We focused our attention on a region (Figure 2) of Nice of $100 \mathrm{~km}^{2}$ (south-east of France: N43 42'41.338" E7 $\left.{ }^{\circ} 15^{\prime} 56.854^{\prime \prime}\right)$. Located between sea and mountains, this area of 350,000 inhabitants is a very heterogeneous area with significant variability of objects. Human construction and vegetation mix. Sparse areas bring out an important plant diversity. There is also the presence of a forest. Different species of palms are present, mainly for ornamental purposes.

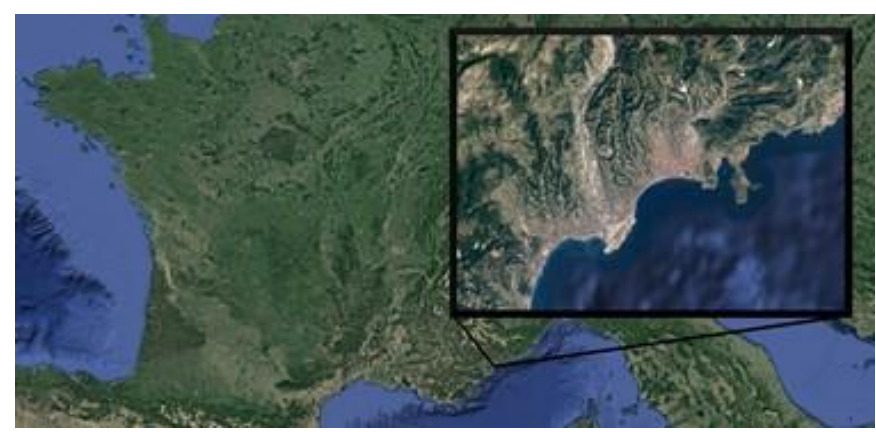

Figure 2. Area of interest

We decided to study in the first place the location of C\&D. Note the important presence of another palm specie, the Washingtonia. In 2014, we estimated approximately 30,000 of $C \& D$ in the area. Since then, a major RPW 
infestation has killed a large part of the Phoenix canariensis (Figure 3 and 4) Images and Data (http://propalmes83.com/index.php/actualites2/144hecatombe-des-palmiers-a-nice-le-procureur-mene-1enquete)

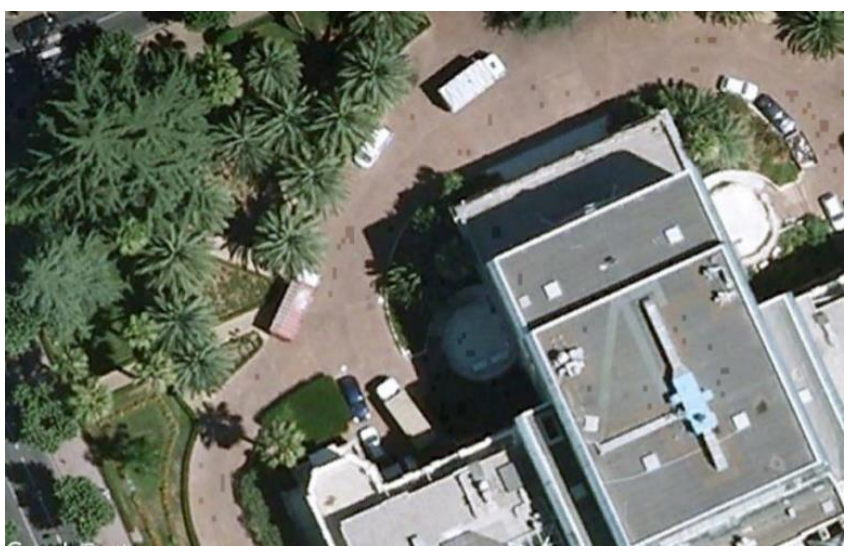

Figure 3. satellite image of palms - Hyères (France) 2007

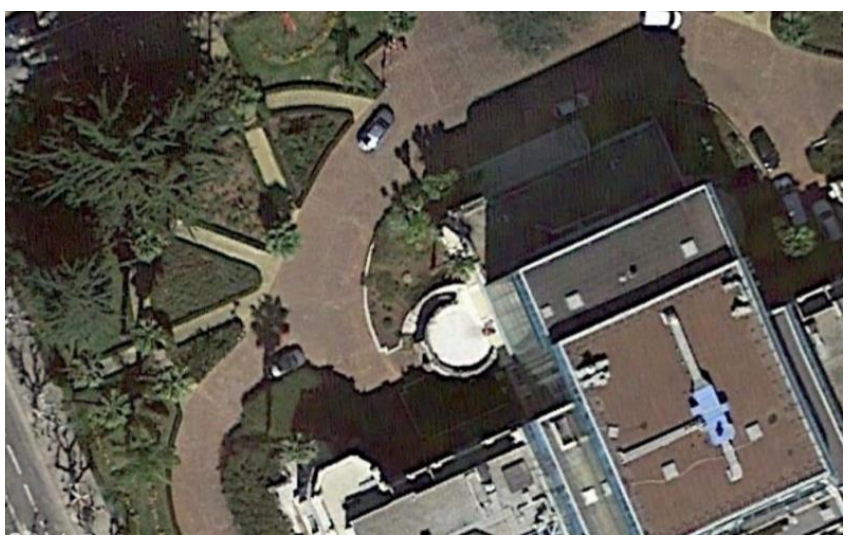

Figure 4. Satellite images of palms Hyères (France) 2018

For this first test, we used Google Maps api images dated 2014, which are free (in limited number and condition), of very high quality in our area of interest $(<30 \mathrm{~cm}$ per pixel), in natural colour RGB ( 8 bytes), and ready to use (there is no need to apply atmospheric correction or image preprocessing). We aggregated 55,000 square images 600 pixels wide to cover the entire area $\left(100 \mathrm{~km}^{2}\right)$.

\section{Filter}

To reduce the cost of treatment, we eliminated obvious nonvegetation areas (such as the sea and large buildings) by applying a simple colour test.

\section{Labelling and set training}

We decided to find C\&D palms in this area. For this purpose, we built a simple GIS browser application (using Node.js and leaflet) to label images (Figure 5). We visually detect and manually selected: (i) 1000 images centred on a palm (a Phoenix canariensis or a Phoenix dactylifera), and (ii) 3000 images of various other objects (including other palms species and containing partial C\&D). For a simple implementation, each image had the same square size of 80 pixel width (around $2.4 \mathrm{~m}$ width at ground level). Then, we artificially enlarged our training set by $90^{\circ}$ rotations (that multiplies the size of sample by 4) and adding a random correction of the brightness (that multiplies the size of sample by 2). So we have 32,000 images to train and test our model.

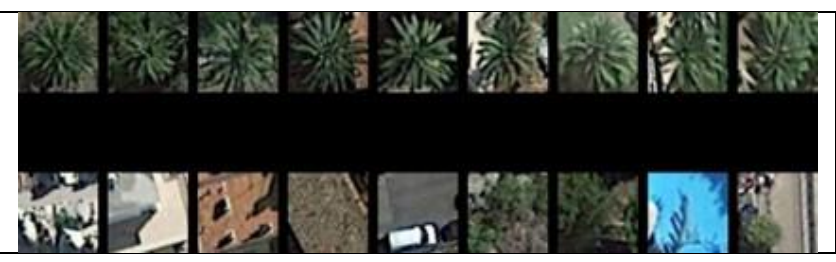

Figure 5. labelled images: C\&D and other objects

\section{Model and architecture}

We built a convolution neural network classifier (Figure 6) with Python and Keras framework. Constituted by: (i) in entry, our 19200 dimension image ( 80 pixels times 80 pixels times 3 colours), (ii) 8 layers of convolution $2 \mathrm{~d}$, ( 5 by 5 kernel) connected with relu activation, (iii) 1 max pooling layer, (iv) 1 full layer with 120 neurons and relu activation, (v) 1 sigmoid activation output.

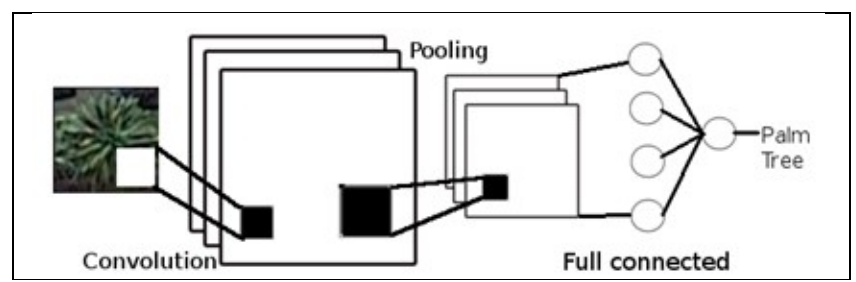

Figure 6. Convolution neural network

\section{Training and testing}

Using our labelled data, we trained our model with binary cross entropy loss and optimizer adam. We used cross validation, training with $90 \%$ of our data and testing with $10 \%$. Stochastic gradient (mini batch) and 2000 epochs allows us to have an F1 score better than $90 \%$ on the test set.

\section{Application to the whole area}

We used our trained model over the whole area using sliding window (Figure 7) (by moving our slider by 5 pixels). At every stage we predicted with our model the confidence of detecting a C\&D palm. We only hold the prediction with a confidence greater than $65 \%$. To avoid overlapping results, we used non-maximal deletion to delete multiple positives from the same object. We recorded the positive results in a database (we used mongodb) with the location (wgs84 coordinates), the confidence index and the number of overlapping images discarded. 


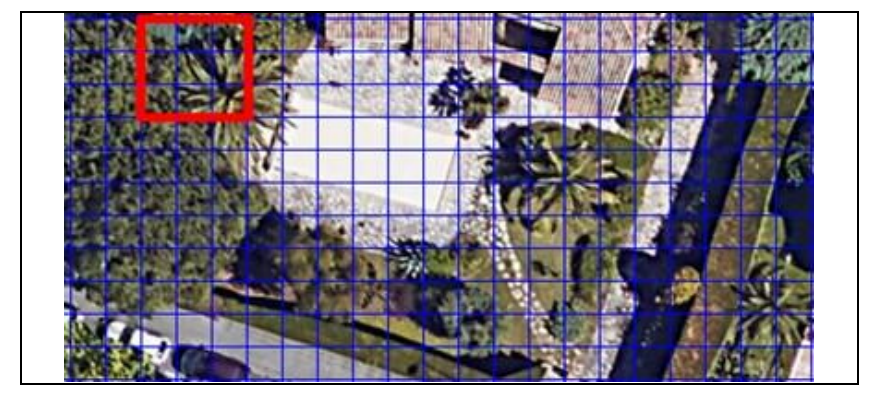

Figure 7. Sliding window

\section{Results}

We located around 20,000 palms in the whole area (Figure 8). In order to evaluate our approach, we manually controlled the results in a smaller but representative area (Figure 9) (around $4 \mathrm{~km}^{2}$ ).

In this area we manually identified and counted around 3000 C\&D. For its part, our model located around 2500 objects, 2000 are C\&D, 500 are false positive (non Phoenix canariensis and non Phoenix dactylifera). Our final metrics had a precision of $79 \%$ (Figure 10), recall 68\% (Figure 11), F1 score $73 \%$ (Figure 12). That means our model located more than $2 / 3$ of the C\&D of the area. And around 1/5 of object found were false positive (non Phoenix canariensis and non Phoenix dactylifera). This evaluation of the results gave an order of magnitude but the exact figures must be taken with care. The control of palms in the area has been done by the human eye, and despite our greater attention, we have to consider a margin of error.

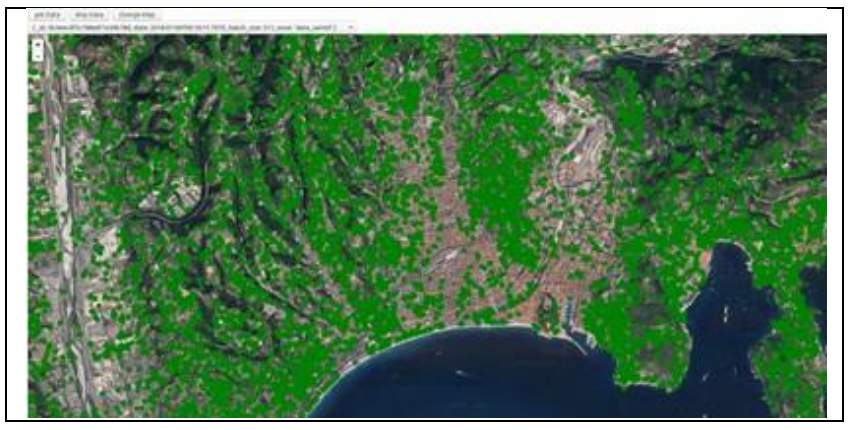

Figure 8. Objects found by the model

We also reviewed the results one by one. We observed that sometimes the algorithm was very efficient, it is able to find a Phoenix canariensis hidden by another tree or in the shadow of a building. But at other times, it totally misses its objective, some shapes found do not look at all like palms. This error can be explained by the classification principle used here, which is one against the other. Which means that the model tried to learn the shapes and colours of specific palms (C\&D), against the shapes and colours of all the other objects. The second group had much more variability. Probably some objects of the second group slipped into the first group because they were not sufficiently represented in the learning dataset. Likewise, the 1,000 C\&D images used for training were selected "at random" by human. We unfortunately tended to select a subsample of the diversity. This significant bias may explain why the model omitted one-third of the objects of interest. It is important to note that the number of Washingtonia found was low, which means that the model was able to understand the difference between different palm species.

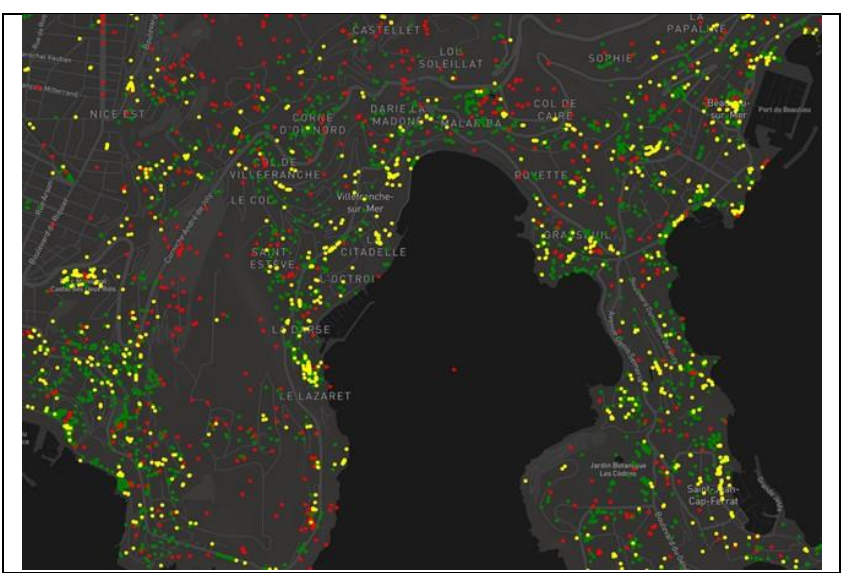

Figure 9. Results evaluation (green $=$ True positive, red $=$ False positive, yellow $=$ False negative)

$$
\text { Precision }=\frac{\text { True Positive }}{\text { True Positive }+ \text { False Positive }}
$$

Figure 10. Precision metric formula

$$
\text { Recall }=\frac{\text { True Positive }}{\text { True Positive }+ \text { False Negative }}
$$

Figure 11. Recall metric formula

$$
\mathrm{F} 1=2 \times \frac{\text { Precision } * \text { Recall }}{\text { Precision }+ \text { Recall }}
$$

Figure 12. F1 score metric formula

\section{Discussion}

The objective of this work is to show the interest of using machine learning (deep learning) and satellite imagery to map palms localization at large scale. Obtaining the exact location of the palms is of great importance for the RPW control programmes. This first results obtained by a simple convolution neural network can be improved by different ways.

Without the need to have 50 million images like the ImageNet database (Krizhevsky, 2012), larger sample of data would represent better the diversity of palms and 
objects, and we would be able to build deeper architecture and train more robust model like the VGG-16 architecture (Simonyan, 2014). Also, the variability of the objects of non interest is very high, it could be easier for the model to learn with multi class than binary class.

Another improvement would be to change the following point: we always used the same size of images, we used sliding windows, and we removed overlapping result. This technique is easy to train but very expensive in calculation. CNN caffe implementation (Yangqing, 2014) or real time detection object detection algorithm (Redmon, 2016) could optimize our method.

It is also interesting to notice that remote sensing, is used to extract more complex information than just detection. Particularly with better resolution imagery obtained by aircraft or UAV, water status of palms for example was studied (Cohen, 2012). Likewise, CNN detection of the palms Raphia in a very dense environment provides better accuracy than human eye (Viennois, 2018). But early RPW detection remains difficult even with UAV. With UAV, interesting results have been obtained, but the infested palms were detected at a late stage (Golomb, 2015). It is not surprising as RPW attack date palms at the trunk level which is different from what occur with the Phoenix canarienis, which is infested at the leaves level. In that case, physical and physiological damages caused by the larvae appear on the foliage that is photographed by satellite or UAV. For the date palms, it is necessary to know that the damages created in the trunk, lead to physiological disorders detectable at the foliage level. This difficulty is well illustrated by the interesting results obtained in the evaluation of the importance of Dubas Bug attacks on date palms by satellite imageries analysis (Al Shidi, 2019). The attack was detectable because the Dubas bug creates direct and indirect symptoms on the foliage. Nevertheless, for the RPW on C\&D late detection would be also very useful, especially in places where the observations of the palms is weak or null.

\section{Conclusion}

This palms detection research using convolution neural networks and satellite imagery in heterogeneous urban area gives encouraging preliminary results. Our evaluation model showed that only $1 / 5$ of the objects found were false positive and more than $2 / 3$ of $C \& D$ were located. Furthermore, various possibilities are available to improve the method: increasing the number of data in order to build and train deeper model. Also, it is possible to enrich the image with other pertinent information provided by satellites (like near infra-red).

It would be interesting to integrate to future research palms infested with RPW, taking into consideration the level of infestation and the difference between date palm and Phoenix canarienisis.

\section{الملخص}

كوزان، رافائيل وميثيل فيري. 2019. التحديد التلقائي لموقع أثجار النخيل بتحليل الصور الفضائية. مجلة وقاية النبات العربية، 37(2): 83-83.

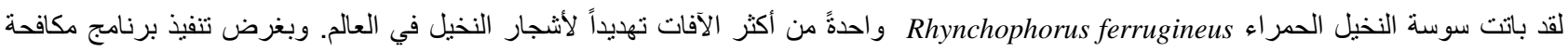

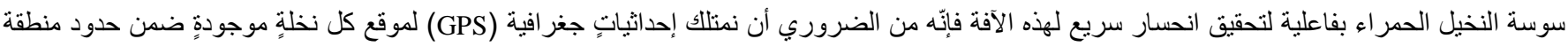

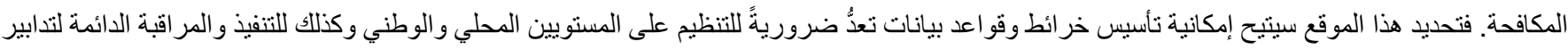

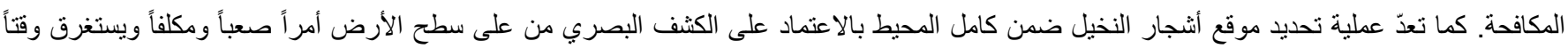

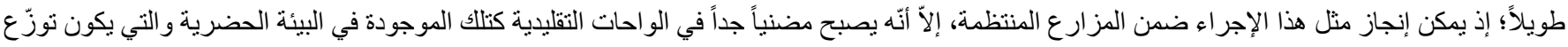

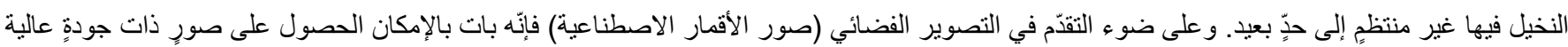

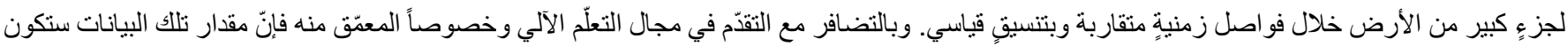

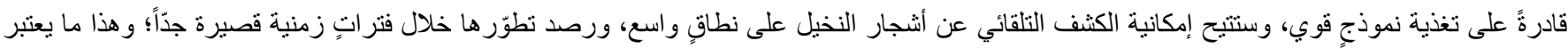

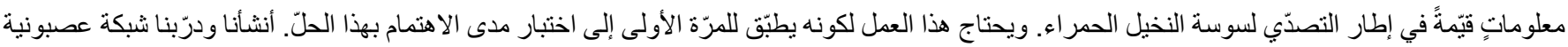

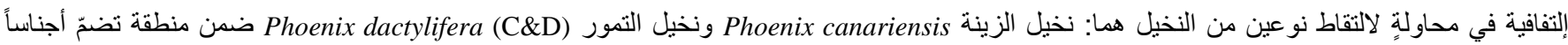

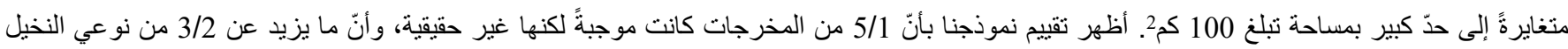

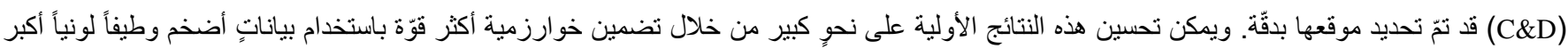

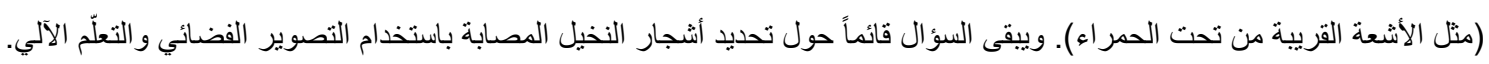

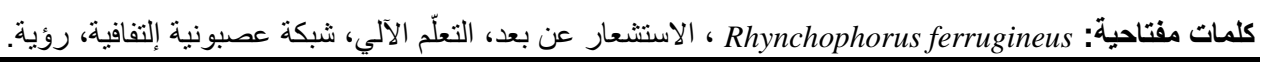




\section{References}

Al Shidi Rashid, H., L. Kumar and S. Al-Khatri. 2019. Detecting Dubas bug infestations using high resolution multispectral satellite data in Oman. Computers and Electronics in Agriculture, 157: 1-11. https://doi.org/10.1016/j.compag.2018.12.037

Cohen, Y., V. Alchanatis, A. Prigojin, A. Levi and V. Soroker. 2011. Use of aerial thermal imaging to estimate water status of palm trees. Precision Agriculture, 13:123-140. https://doi.org/10.1007/s11119-011-9232-7

Fajardo, M., X. Rodríguez, C.D. Hernández, L. Barroso, M. Morales, A. González and R. Martín. 2017. The Canary Island success story in eradicating red palm weevil. Proceedings "Scientific Consultation and High-Level Meeting on Red Palm Weevil Management", organized by FAO and CIHEAM, Italy, 29-31 March, 2017, Rome, Italy

Faleiro, J.R., M.A. El-Saad and A.H. Al-Abbad. 2011. Pheromone trap density to mass trap Rhynchophorus ferrugineus (Coleoptera: Curculionidae/ Rhynchophoridae/ Dryophoridae) in date plantations of Saudi Arabia. International Journal of Tropical Insect Science, 31: 75-77.

FAO. 2017 Rome declaration on RPW. Presented at the "Scientific Consultation and High-Level Meeting on Red Palm Weevil Management", organized by FAO and CIHEAM, Italy, 29-31 March, 2017, Rome, Italy

Ferry, M. and S. Aldobai. 2017. National components of the strategy for the management of red palm weevil. Presented at the "Scientific Consultation and HighLevel Meeting on Red Palm Weevil Management", organized by FAO and CIHEAM, Italy, 29-31 March, 2017, Rome, Italy

Golomb, O., V. Alchanatis, Y. Cohen, N. Levin and V. Soroker. 2015. Detection of suspected trees infected with Red Palm Weevil by thermal imaging. Precision agriculture, 15: 643-650. https://doi.org/10.3920/978-90-8686-814-8_80

Krizhevsky, A., I. Sutskever and G.E. Hinton. 2012. ImageNet Classification with Deep Convolutional Neural Networks. NIPS'12 Proceedings of the 25th International Conference on Neural Information Processing Systems, 1: 1097-1105. https://doi.org/10.1145/3065386

Labrador-García, M., A. Alonso, S. Idbraim, M. Arbelo, L. Núñez, A. Lorenzo and J.A. Guerra. 2013. Cartografiado de palmeras en la región de souss massa draa (marruecos) mediante imágenes del satélite WorldView-2.
LeCun, Y., Y. Bengio and G. Hinton. 2015. Deep learning. Nature, 521: 436-444. https://doi.org/10.1038/nature14539

Li, W., H. Fu, L. Yu and A. Cracknell. 2016. Deep learning based oil palm tree detection and counting for highresolution remote sensing images. Remote Sensing, 9: 22 https://doi.org/10.3390/rs9010022

Oehlschlager, A.C. 2006. Mass trapping and strategy for management of Rhynchophorus palm weevils, pp. 143 168. In Proceedings of I Jornada Internacional sobre el Picudo Rojo de las Palmeras, November 2005, Valencia, Spain. Fundacion Agroalimed-Generalitat Valenciana, Valencia, Spain

Redmon, J., S. Divvala, R. Girshick. and A. Farhadi. 2016. You Only Look Once: Unified, Real-Time Object Detection. Pp. 779-788 In: Proceedings of The IEEE Conference on Computer Vision and Pattern Recognition (CVPR). https://doi.org/10.1109/CVPR.2016.91

Santoso, H., H. Tani and X. Wang. 2016. A simple method for detection and counting of oil palm trees using highresolution multispectral satellite imagery. International journal of remote sensing, 37: 5122-5134. https://doi.org/10.1080/01431161.2016.1226527

Simonyan, K. and A. Zisserman. 2014. Very deep convolutional networks for large-scale image recognition. arXiv technical report

Soroker, V., D. Blumberg, A. Haberman, M. Hamburger-Rishard, S. Reneh, S. Talebaev, L. Anshelevich and A. R. Harari. 2005. Current status of red palm weevil infestation in date palm plantations in Israel. Phytoparasitica, 33: 97-106 https://doi.org/10.1007/bf02980931

Viennois Gaëlle, Borne Frédéric, Jaeger Marc, Borianne Philippe. 2018. Quelle vérité terrain pour les réseaux de neurones en imagerie drone ? Application à la détection de palmier Raphia en forêts au Gabon.Conférence Française de Photogrammétrie et de Télédétection, Marne-la-Vallée, France, 26 June 2018/27. SFPT, 3p.

Yangqing, Jia, E. Shelhamer, J. Donahue, S. Karayev, J. Long, R. Girshick, S. Guadarrama and T. Darrell. 2014. Caffe: Convolutional Architecture for Fast Feature Embedding. Pages 675-678. In: Proceedings of the 22nd ACM international conference on Multimedia, November 03-07, 2014, Orlando, Florida, USA. https://doi.org/10.1145/2647868.2654889 